\title{
The Forerunner UAV Concept for the Increased Safety of First Responders
}

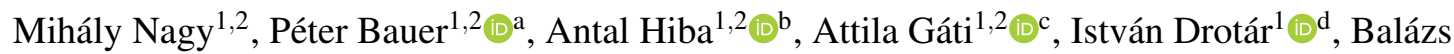 \\ Lattes $^{3}$, Ádám Kisari ${ }^{1,2}$ (D) \\ ${ }^{1}$ Institute of Problem Solving, Széchenyi István University, Egyetem tér 1, Györ, Hungary \\ ${ }^{2}$ Institute for Computer Science and Control, Budapest, Hungary \\ ${ }^{3}$ National Media and Infocommunications Authority, Budapest, Hungary \\ nagymihaly@sztaki.hu,bauer@sztaki.hu,hiba.antal@sztaki.hu,drotar.istvan@sze.hu
}

Keywords: Forerunner UAV, First responders, Basic concepts, Wireless communication

Abstract: $\quad$ This paper proposes a novel Forerunner UAV concept to increase the safety of first responders by monitoring the road in front of their emergency ground vehicle (EGV) and notifying the driver about any violation of his/her right of way or approaching danger. The developments are conducted in an R\&D project in Hungary. The proposed UAV for the planned urban demonstration is a hexacopter with triple redundant architecture applying a gimbaled camera to monitor the surroundings. In the cooperative control of the EGV and UAV the UAV must fly in front of the EGV which is possible through wireless communication of route data and velocity. Besides the real system a computer simulation representation is also applied including CARLA and Matlab to make exhaustive tests of the system capabilities. Increased attention is devoted to the possible wireless communication solutions as these are safety critical parts of the system. The article ends with the lists of planned simulation and real test scenarios to evaluate the system.

\section{INTRODUCTION}

Considering the overview works and statistics in the US a major cause of the injury and death of first responders besides injuries and deaths in action is due to traffic incidents (crashes) see (Casey C. Grant and Merrifield, 2011), (Donoughe et al., 2012), (NHTSA, 2014), (Smith, 2015). Unfortunately we could not find any EU statistics but accidents involving first responders regularly happen in Hungary. In May 2019 there was a crash in the US (luckily with only minor injuries) caught on video between a train and a police car $(\mathrm{CNN}$, 2019) (https://edition.cnn.com/videos/us/2019/05/ 22/train-hits-police-cruiser-newsource-orig.cnn) because the police car did not see the approaching train behind the other. An aerial view of the situation could help the driver to avoid this accident as possibly could help in several situations when the approaching

\footnotetext{
a (iD https://orcid.org/0000-0000-0000-0000

b (iD https://orcid.org/0000-0000-0000-0000

c (D) https://orcid.org/0000-0000-0000-0000

d (iD https://orcid.org/0000-0000-0000-0000

e (iD https://orcid.org/0000-0000-0000-0000
}

vehicle is covered from the driver. This has led us to the idea to propose a Forerunner UAV (unmanned aerial vehicle) which can fly in front of the emergency ground vehicle (EGV) and monitor the road / area to detect approaching vehicles and vehicles failing to give the right of way. In case of hazard detection, the driver should decrease speed and handle the situation. Beyond risk alert, the minimum requirement is to stream the aerial view to the driver of the EGV to increase his/her situational awareness. Further improvements can be to colour the area in front of the vehicle (green $=$ free route, red $=$ danger, not enough space to pass) and/or denote the threatening vehicle(s) with red colour.

To the best of our knowledge, this Forerunner UAV name has not been used in this meaning, searching for forerunner drone/uav/aircraft/helicopter in Google Chrome the best hits are:

- The forerunner position of a drone company (Inside Unmanned Systems, 2019)

- A computer company (Forerunner Computer Systems, 2020)

- A computer game character (fandom.com, 2020) 


\section{- A sportwatch (Garmin, 2020)}

so the Forerunner UAV name (and the concept) can be considered completely new. This year (2020) a research project is started in Hungary to evaluate the feasibility of the Forerunner UAV concept and this article is the first publication summarizing the initial findings of the development.

Considering the literature about following and/or supporting a ground vehicle $(\mathrm{GV})$ by an aerial vehicle several solutions can be found. First, there are solutions helping the remote operator of the GV with the aerial images taken by the UAV on demand of the operator (Cantelli et al., 2013) or with continuously streamed video (Harik et al., 2015). Second, solutions utilizing aerial images in pre-planning of $\mathrm{GV}$ route (Zoto et al., 2020) can be found. Third, there are solutions on-line supporting the unmanned ground vehicle (UGV) in path planning by processed information from the UAV images (free paths, obstacles etc.) (Lakas et al., 2018), (Peterson et al., 2018). Another related source discusses the tracking of an UGV by a UAV considering changes in UGV speed and heading but constant UAV speed (Lee et al., 2003).

Considering the forerunner concept here the UAV needs to fly in front of the EGV to have images about forthcoming obstacles or threatening vehicles in advance. As nowadays the emergency vehicle drivers navigate by their own GPS and probably they suddenly modify the route (especially in intersections) based on their knowledge about the area its advisable to plan and follow the route on-board the EGV and follow the EGV with the UAV. So from the above literatue review the following of the $\mathrm{GV}$ by the UAV (Lee et al., 2003) and the support of the GV operator (who is on-board the EGV in this case) through streamed video (Harik et al., 2015) are the closest to our concept but variable UAV speeds should be considered and the UAV must fly in front of the EGV.

This paper is an introduction of the basic concepts considered in the design and development of the Forerunner UAV solution. Section 2 summarizes the hardware system and the ideas for the cooperative control concept between the EGV and UAV. Section 3 summarizes the computer simulation concept of the overall forerunner system including the EGV, the UAV, the camera sensor model and the related virtual 3D environment. Section 4 deals with the communication issues regarding position and route data communication between the EGV and UAV and the streaming of video from the UAV to the EGV. Section 5 gives an overview about the planned simulation and real life test scenarios to evaluate the pros and cons of the forerunner concept and demonstrate its basic capabilities and finally Section 6 concludes the paper.

\section{SYSTEM CONCEPT}

In our approach, the UAV supplements the EGV to extend its sensing capabilities. The aerial vehicle has to be autonomous and has to follow or get ahead of the EGV as the circumstances require. For example if the EGV has to go through a crowded city center, it is advised for the drone to stay ahead relatively close to the EGV in order to inspect the upcoming intersections. On the other hand, in a highway or motorway scenario the drone can get further ahead to identify obstacles, providing the driver with extra time to safely reduce speed.

Both of these cases prove that the UAV is not necessarily following its companion rather leading the group. However, the EGV is the one that dictates the route. This problem can be solved if the UAV knows the proposed path of the EGV. Therefore, at the start of the journey, the destination, the planned route and the initial course has to be sent to the drone. Of course, this is not a final decision the EGV can alter its route due to heavy traffic or other reasons, so the UAV has to adapt to the emergency vehicle whenever it is necessary. To achieve this, the drone is commanded to stop at every intersection and wait for the emergency vehicle, meanwhile scanning the environment for possible hazards. After it is evident which way the EGV is going (from communication or the motion of the EGV) the drone starts to go to the same direction.

To be able to observe the surroundings of the emergency vehicle, the UAV has to be equipped with a camera preferably attached with a gimbal to enable independent movement relative to the drone. The whole concept relies on the perception of the camera, therefore it has to be carefully selected. The image processing is highly dependent on the point of view of the camera. Its task is to identify pedestrians and vehicles and their position on the ground. The current state of development suggests, that bounding box (YOLO) based identification could be carried out real-time on the computer aboard the drone.

From the above concept it is obvious that there has to be some kind of communication between the UAV and the EGV. Not only the planned route and course has to be sent at the beginning but during movement several data (including velocity and change of plans) must be transmitted from the ground to the aerial vehicle serving as the base of the cooperative control. On the other hand, the UAV sends the risk alerts, raw and/or (pre-)processed camera image to the ground to help the driver. The place where image processing is carried out is going to be determined by the performance of the on-board computer 
of the drone. On-board image processing would pose smaller challenge to the inter-vehicle communication because post-processed images are much less data. The communication issues are discussed in detail in Section 4.

After the overall concept of the Forerunner UAV system is described our proposed hardware scheme is shown in Fig. 1.

As it can be seen in Fig. 1 our drone choice is the DJI M600 Pro. The reason behind it is that it offers redundancy on multiple levels, for example it features six batteries, three sets of IMU sensors and six rotors. This makes the device safer to fly, which is a key feature when using it in possibly life-threatening scenarios and also key for the approval of airspace authorities. The drone is equipped with the DJI A3 autopilot system which will be completed with an onboard computer the DJI Manifold 2 which is responsible for the high-level control and data collection from the RTK GPS unit (SCL RTK in the figure) and camera module. Furthermore, the communication with the ground vehicle is also carried out by the Manifold 2 computer.

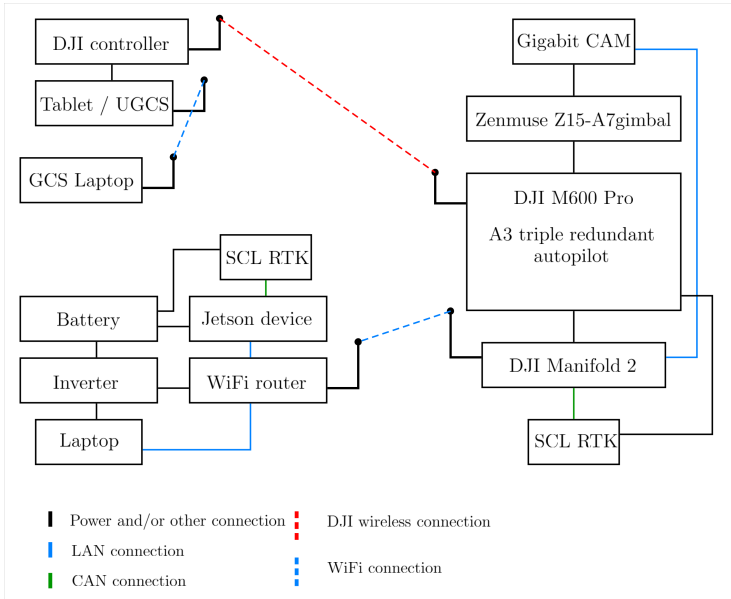

Figure 1: Hardware concept of the Forerunner UAV system

Considering the drone choice a serious game stopper in the Forerunner UAV concept can be the limited speed range of the available drones that is why an extensive search was done to have an overview about the possibilities. The results are summarized in Table 1 (VTOL means vertical take-off and landing). As the table shows most of the drones are only capable to reach about $90 \mathrm{~km} / \mathrm{h}$ maximum speed which can be satisfactory in urban environment but is not enough on country roads and freeways. However, the Schiebel Camcopter's $220 \mathrm{~km} / \mathrm{h}$ maximum speed makes the concept feasible in the future even on freeways. We also assume that drone industry continues to produce better and so faster drones in near future.

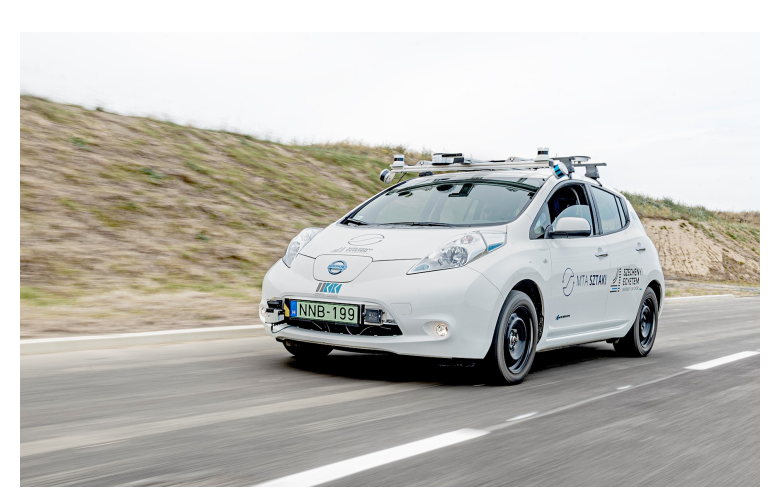

Figure 2: Nissan Leaf experimental ground vehicle

Using a proper emergency response vehicle during development and testing would be cumbersome so we plan to employ our Nissan Leaf (see Fig. 2) test vehicle as a substitute. The regular passenger car has now been heavily modified to become an autonomous vehicle test platform (Somogyi et al., 2018). It is currently mounted with several sensors such as LIDARs, a radar, cameras, and high precision navigation sensors. It is also equipped with several computation units to handle data and control tasks. Though it is capable of autonomously navigating we plan to use it in human-driven mode as the ground vehicle of the group while also taking advantage of the already operational navigation system, on-board processing, and user interface devices. However, as Fig. 1 shows we also plan to develop a standalone ground segment with battery, board computer, RTK GPS and communication module to be able to use it on any human driven vehicle replacing the Leaf during the developments.

\section{SIMULATION CONCEPT}

As the real scenario testing capabilities are very limited due to the availability of the proving ground $\mathrm{Za}$ lazone (zalazone.hu/en) and they require much more effort we only plan to show system capabilities in low speed urban maneuvers (see Section 5). That's why an extensive simulation test campaign is very important to evaluate the possible capabilities of the Forerunner UAV concept in all situations (see Section 5). 
Table 1: Drone types with maximum cruise speed

\begin{tabular}{|c|c|c|}
\hline Structure & Type & Max. speed $[\mathrm{km} / \mathrm{h}]$ \\
\hline VTOL & Quantum Systems Vector & $54-90$ \\
\hline VTOL & Quantum Systems Tron F90+ & 64.8 \\
\hline VTOL & UKR Spec Systems PD-1 & 90 \\
\hline VTOL & L3Harris FVR-90 & 92.6 \\
\hline VTOL & Blackswift S2 & 64.8 \\
\hline \hline Quadcopter & DJI M300 & 82 \\
\hline Quadcopter & DJI Inspire 2 & 94 \\
\hline Quadcopter & Freefly Alta X & 95 \\
\hline \hline Hexacopter & DJI M600 Pro & 64 \\
\hline Hexacopter & Yuneec Typhoon H & 70 \\
\hline \hline Helicopter & Schiebel Camcopter S-100 & 220 \\
\hline
\end{tabular}

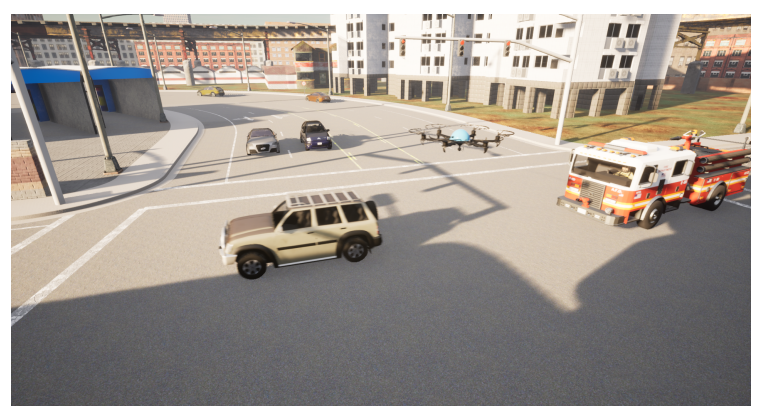

Figure 3: Fire truck with forerunner drone in intersection

A realistic forerunner drone simulation (see Fig. 3) requires a drivable EGV, a drone with simulated camera sensor, and an environment with other vehicles and pedestrians. CARLA simulator (Dosovitskiy et al., 2017) with Unreal4 game development engine provides a functional city with programmed intersections, vehicle and walker AI (artificial intelligence) agents, detailed simulated sensors (RGB camera, depth camera, LIDAR etc.) and a python API. Into this solid basis we integrate our forerunner UAV with the on-board camera system, our test scenarios with other vehicles, and our image processing and decision making logic. We plan to drive the EGV with game controllers to make demonstrations more interesting by involving the bystanders to drive the vehicle. The dynamics of the Forerunner UAV is simulated in Matlab (visulaization done in CARLA) and an UDPbased communication (similar to the real system) is implemented between the Matlab (UAV) and CARLA (EGV) part (see Fig. 4).

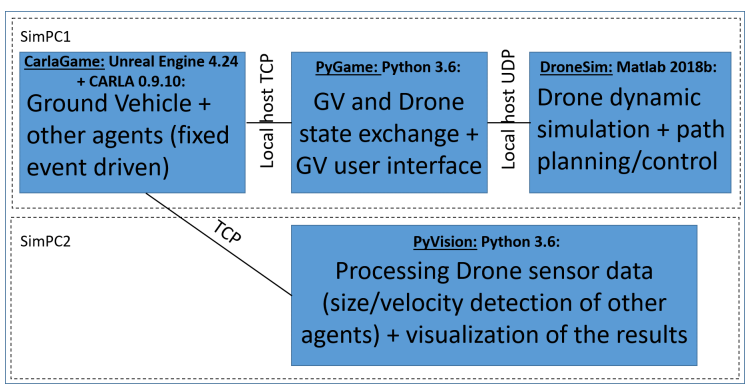

Figure 4: Main components of the simulator and their tasks

Fig. 4 summarizes the main components of our simulator. The basis of the simulator is CARLA which can be modified in Unreal editor. A firetruck is added as a version of an existing drivable truck in CARLA, while the drone is a base vehicle pawn, which can be manipulated through the python API according to our own Matlab simulation. PyGame part is responsible for the user interface of the driver (steering wheel or keyboard). PyVision is our image processing and decision making module, which can run on a separate computer.

The Matlab Simulink UAV simulation incorporates detailed attitude and position dynamics, modeling of the propulsion system and the implemented control method. The control loop uses the incoming data from the EGV (velocity and planned route) to be able to maintain a certain distance in front of it. After calculating the displacement and heading of the drone, the data is sent to the CARLA simulation which then draws the model accordingly. The parameterization of the drone model is done to represent a similar model to the DJI M600 Pro planned to be applied during the real life tests of the Forerunner UAV system.

The drone should detect other vehicles, cyclists and pedestrians to decide if the EGV can pass the intersection at high speed or not. To do this, a 3D se- 
mantic map of the neighborhood of the intersection should be generated from camera images. The simulation environment will also give us the possibility to develop gimbal management strategy(s) for the monitoring of the intersection, and to define the required time and frame rate for decision on the safe EGV pass.

The first version of our image processing is based on a semantic segmentation of drone camera according CityScapes concept (Cordts et al., 2016). The output of the simulator is not given back to the PyGame module because we will possibly send only decisions to the EGV from the UAV if the wireless communication capabilities are too limited. However, we plan to stream the video to the EGV and it is possible that processed images will also be sent to the EGV to give more insight in the case of a stalled situation.

After simulation testing in urban scenarios country road and freeway scenarios will be implemented and tested as listed in Section 5.

\section{COMMUNICATION ISSUES}

As stated before, the communication between the UAV and the EGV will be crucial in the success of the Forerunner UAV system that is why extensive comparison of possible solutions and even communication system tests are conducted in this early stage of the project.

One of the best possible solutions is to establish a connection according to the IEEE 802.11 standard. Nowadays these systems work on the 2400-2483.5 $\mathrm{MHz}, 5150-5875 \mathrm{MHz}$, and $57-71 \mathrm{GHz}$ frequency bands. The devices that use 802.11 protocol for communication are simple radio units, but these systems are robust and interference tolerant.

The standard uses the ISM frequency bands for communication. The ISM bands are free to use, so the operation on these frequencies is only limited by the regional legislation. Furthermore, the 802.11 standard's additional advantage is the $15-20 \mathrm{~ms}$ typical response time (this is the minimal expected limit).

The popularity of the standard is shown by the fact that throughout the years several versions of the original standard has been published. The new standards introduced new frequency band usage, higher data rate, and more robust modulations. The current newest standard is the Wi-Fi 6, which is described in the 802.11ax standard. Table 2 shows the data rate calculation for $802.11 \mathrm{ac}$ standard.

Values shown in the table are not the maximum data rates that the standard describes (256-QAM). The selection was based on several factors, like the phase distortion caused by motion, and destructive or additive interference according to the phase difference of multipath signals. As for the code ratio, the same pattern can be seen. The chosen values are more robust than the highest ratio of the standard. Regarding the guard interval (GI) the table has calculations for $400 \mathrm{~ns}$ and $800 \mathrm{~ns}$ as well. Considering the application, the higher $800 \mathrm{~ns}$ values can guarantee the fail-safe functionality in an environment with reflections. The calculations for the video signal should be evaluated by these factors.

The Forerunner UAV application can apply two possible variations:

- FHD video signal streaming to a vehicle, where the post-processing happens;

- The post-processing happens on-board of the $\mathrm{UAV}$, and the results are being transferred to the vehicle in an array.

In the first situation, calculating with the display ratio (1920x1080) and using the H.264 (HVC) video compression, $8.5 \mathrm{Mb} / \mathrm{s}$ data rate can be estimated. While with the H.265 (HEVC) compression only 4.4 $\mathrm{Mb} / \mathrm{s}$ for a 30 frame/sec video stream.

In the second version, the display format should be at least 4 CIF which is $704 \times 480$ pixel, with the application of H.264 compression there should be around $1.6 \mathrm{Mb} / \mathrm{s}$ regarding the data rate. Meanwhile the H.265 method should provide the average 0.8 $\mathrm{Mb} / \mathrm{s}$ data rate.

For the calculations, the urban areas are the most critical (see Fig. 5). In these areas where the device distributions are very dense, the proper performance must be guaranteed. The interference in these areas is significantly higher and the systems must change to less crowded frequency band (for stable data transmission) even in a $100 \mathrm{~m}$ radius. In case of Active Scan, it needs higher data rate (min. $4 \mathrm{Mb} / \mathrm{s}$ ) for a $30 \mathrm{~ms}$ scanning every 5 second. If the vehicle moves with $50 \mathrm{~km} / \mathrm{h}$ there will be around $70 \mathrm{~m}$ distance between two measurement.

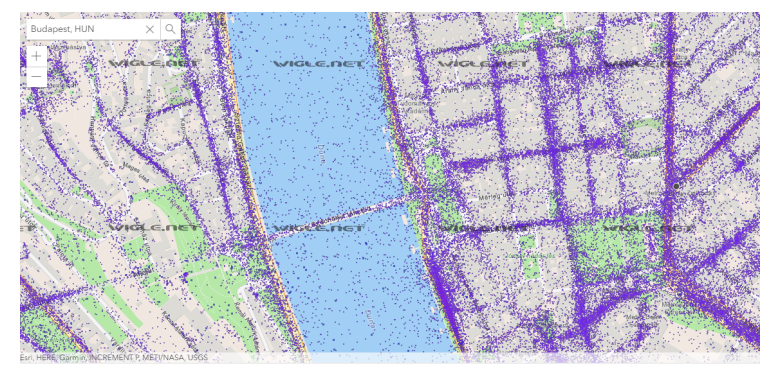

Figure 5: Number of Access Points in Budapest (bobzilla et al., 2020)

Another problem regarding the 802.11 standard, is that the devices can order more users to the avail- 
Table 2: Maximum theoretical data rates

\begin{tabular}{|c|c|c|c|c|c|c|}
\hline \multirow{3}{*}{ Spatial stream } & \multirow{3}{*}{ Modulation } & \multirow{3}{*}{ Code ratio } & \multicolumn{4}{|c|}{ Maximum theoretical data rate } \\
\hline & & & \multicolumn{2}{|c|}{$20 \mathrm{MHz}$ channel } & \multicolumn{2}{|c|}{$40 \mathrm{MHz}$ channel } \\
\hline & & & GI $800 \mathrm{~ns}$ & GI $400 \mathrm{~ns}$ & GI $800 \mathrm{~ns}$ & GI $400 \mathrm{~ns}$ \\
\hline 1x1 SISO & 16-QAM & $1 / 2$ & $26 \mathrm{Mb} / \mathrm{s}$ & $28.9 \mathrm{Mb} / \mathrm{s}$ & $54 \mathrm{Mb} / \mathrm{s}$ & $60 \mathrm{Mb} / \mathrm{s}$ \\
\hline $1 \times 1$ SISO & 16-QAM & $3 / 4$ & $39 \mathrm{Mb} / \mathrm{s}$ & $43.3 \mathrm{Mb} / \mathrm{s}$ & $81 \mathrm{Mb} / \mathrm{s}$ & $90 \mathrm{Mb} / \mathrm{s}$ \\
\hline $1 \times 1 \mathrm{SISO}$ & 64-QAM & $2 / 3$ & $52 \mathrm{Mb} / \mathrm{s}$ & $57.8 \mathrm{Mb} / \mathrm{s}$ & $108 \mathrm{Mb} / \mathrm{s}$ & $120 \mathrm{Mb} / \mathrm{s}$ \\
\hline $1 \times 1 \mathrm{SISO}$ & 64-QAM & $3 / 4$ & $58.5 \mathrm{Mb} / \mathrm{s}$ & $65 \mathrm{Mb} / \mathrm{s}$ & $121.5 \mathrm{Mb} / \mathrm{s}$ & $135 \mathrm{Mb} / \mathrm{s}$ \\
\hline $2 \times 2$ MIMO & 16-QAM & $1 / 2$ & $52 \mathrm{Mb} / \mathrm{s}$ & $57.8 \mathrm{Mb} / \mathrm{s}$ & $108 \mathrm{Mb} / \mathrm{s}$ & $120 \mathrm{Mb} / \mathrm{s}$ \\
\hline $2 \times 2$ MIMO & 16-QAM & $3 / 4$ & $78 \mathrm{Mb} / \mathrm{s}$ & $86.7 \mathrm{Mb} / \mathrm{s}$ & $162 \mathrm{Mb} / \mathrm{s}$ & $180 \mathrm{Mb} / \mathrm{s}$ \\
\hline $2 \times 2$ MIMO & 64-QAM & $2 / 3$ & $104 \mathrm{Mb} / \mathrm{s}$ & $115.6 \mathrm{Mb} / \mathrm{s}$ & $216 \mathrm{Mb} / \mathrm{s}$ & $240 \mathrm{Mb} / \mathrm{s}$ \\
\hline $2 \times 2$ MIMO & 64-QAM & $3 / 4$ & $117 \mathrm{Mb} / \mathrm{s}$ & $130.3 \mathrm{Mb} / \mathrm{s}$ & $243 \mathrm{Mb} / \mathrm{s}$ & $270 \mathrm{Mb} / \mathrm{s}$ \\
\hline
\end{tabular}

able spectrum. The regulations define three layers of users. The second- and third-layer devices cannot interfere with the first layer applications. This applies to the second- and third-layer's relationship too. In conclusion the third layer devices cannot cause any interference to the first- and second-layer systems. The previously mentioned applications in the 2400-2483.5 $\mathrm{MHz}$ and $5150-5875 \mathrm{MHz}$ frequency bands are third layer devices.

The other possibility is the civilian MFCN network with LTE and NR services. These technologies have similar or lower response time than the 802.11 systems. For the LTE connections the average response time is around 10-15 ms. The 5G NR system's can be $1 \mathrm{~ms}$ (NOKIA, 2016) depending on the application. These particular systems work with wider range of frequency bands $(700 \mathrm{MHz}, 800 \mathrm{MHz}, 1800 \mathrm{MHz}$, $2100 \mathrm{MHz}, 3,5 \mathrm{GHz}, \ldots$ ) where they have priority channel utilization. The above-mentioned bands are dedicated for a specific service provider. In this situation the placements of base stations are limited to more occupied areas, like cities and roads. In rural areas only the UMTS network can be ensured, which cannot provide the appropriate values for the communication.

Overall, the urban areas are suitable for MFCN (or not civilian PPDR and BBDR) system usage, and rural areas are convenient for the 802.11 networks.

A preliminary test was also run to determine the latency of wireless image transmission between two hosts. IEEE 802.11n compliant devices were used operating in the $5 \mathrm{GHz}$ mode with the use of two spatial streams at a channel width of $40 \mathrm{MHz}$. The sender was connected to a Cisco E2000 wireless router via WLAN, while the receiver communicated with the router on Gigabit ethernet. Using the 64-QAM modulation scheme the standard provides a $300 \mathrm{Mb} / \mathrm{s}$ maximum theoretical data rate. In our case an effective data rate of $115 \mathrm{Mbit} / \mathrm{s}$ was measured. We applied NewTek's NDI API to produce video streams both in low and high resolutions. In both cases the frame rate was 20 fps. Table 3 shows the results.

Table 3: Latency of Video Transmission Tests

\begin{tabular}{|c|c|}
\hline Resolution & Latency \\
\hline $640 \times 480$ & $30 \mathrm{~ms}$ \\
\hline $1440 \times 1080$ & $40 \mathrm{~ms}$ \\
\hline
\end{tabular}

Table 4: List of Abbreviations

\begin{tabular}{|l|l|}
\hline Abbreviation & Definition \\
\hline 4CIF & $\begin{array}{l}\text { 4xCommon Intermediate For- } \\
\text { mat }\end{array}$ \\
\hline AP & Acces Point \\
\hline BBDR & $\begin{array}{l}\text { Broadband Public Protection } \\
\text { and Disaster Relief }\end{array}$ \\
\hline FHD & Full High Definiton \\
\hline GI & Guard Intervall \\
\hline LTE & Long Term Evolution \\
\hline MFCN & $\begin{array}{l}\text { Mobile/Fixed Communication } \\
\text { Network }\end{array}$ \\
\hline MIMO & Multiple Input Multiple Output \\
\hline NR & New Radio \\
\hline PPDR & $\begin{array}{l}\text { Public Protecion and Disaster } \\
\text { Relif }\end{array}$ \\
\hline QAM & $\begin{array}{l}\text { Quadrature Ampltide Modula- } \\
\text { tion }\end{array}$ \\
\hline SISO & Single Input Single Output \\
\hline WLAN & Wireless Local Area Network \\
\hline
\end{tabular}

\section{TARGETED TEST SCENARIOS}

The goal of the simulation campaign is to extensively test the capabilities of a possible forerunner system considering scenarios from urban to freeway as first responders can operate in any of these scenarios. The planned test scenarios and situations are: 
- EGV approaches an intersection with traffic light (red to the EGV) or priority to the right and crosses straight. Car coming from right stops or not.

- EGV approaches an intersection with traffic light (red to the EGV) or priority to the right and turns left. Car coming from right and/or front stops or not.

- EGV approaches a crowded intersection with traffic light (red to the EGV) or priority to the right and crosses straight. Cars before the crossroad get out of the way except for one and/or car coming from right stops or not.

- EGV approaches a roundabout where other vehicles stop to give way or not.

- EGV drives with high speed on a country road, other vehicles give way on the road and at crossroads or not.

- EGV drives with high speed on a curved country road, other vehicles give way or not.

- EGV approaches a slow vehicle on a country road is it possible to overtake or not?

- EGV drives on a freeway, other vehicles give way or not, vehicle with technical breakdown causes a problem.

The goal of the real demonstration is to show the basic capabilities of a Forerunner UAV system. Considering the features of the targeted proving ground Zalazone (zalazone.hu/en) this demonstration is planned in a low speed artificial urban environment, the so called Smart City. The planned scenarios are:

- EGV approaches an empty intersection with priority to the right and crosses it straight.

- EGV approaches an intersection with priority to the right and crosses it straight. Car coming from right stops, EGV continues.

- EGV approaches an intersection with priority to the right and crosses it straight. Car coming from right does not stop, EGV must brake.

- EGV approaches an empty intersection with priority to the right and turns left.

- EGV approaches an intersection with priority to the right and turns left. Other vehicles coming from front and/or right all stop, EGV continues.

- EGV approaches an intersection with priority to the right and turns left. Other vehicles coming from front and/or right do not stop all, EGV must brake.

\section{CONCLUSIONS}

This paper introduces the Forerunner UAV concept which to the best of the authors knowledge is a new idea . It includes an UAV equipped with an on-board camera flying in front of the emergency ground vehicle (EGV) and checking if its right of way is provided or not. It can notify the driver, stream the video to the driver of the EGV and in improved versions can also highlight the dangerous vehicles / spots on the video image. This can decrease the risk of traffic accidents involving EGVs and so increase the safety of first responders.

After presenting this concept the article introduces the main topics discussed during the early development of the system and the findings of these discussions.

The overall system concept is based-on the cooperative control of the EGV and UAV where the UAV should fly in front of the EGV knowing its planned route but stopping and waiting for it in every intersection to be able to adapt to sudden route changes. The DJI M600 Pro hexacopter was selected as the UAV because of its triple redundant architecture and payload capacity. The demonstration ground vehicle is planned to be the Nissen Leaf vehicle of the research group but an independent ground segment will be designed which can be easily installed on any ground vehicle with proper load capacity.

As the planned real demonstrations will cover only a limited subset of the required capabilities of the system an extensive simulation test campaign is planned to evaluate all of the capabilities. This is built on the CARLA simulator with Unreal engine simulating the EGV and its environment. The UAV is simulated in Matlab Simulink and an UDP protocol-based communication similar to the real one provides the connection between them. Simulated camera images are generated in CARLA and the image processing is planned to be done on a separate computer.

As the communication of planned route and the video stream between the EGV and UAV is a crucial safety critical part of the system a separate section is devoted to the communication possibilities and system tests focusing on data rates and delays.

Finally, the last section summarizes the planned simulation and real demonstration scenarios attempting to cover the whole range of possible situations.

Future work includes the selection of the communication devices and protocols, the detailed design, tuning and testing of the cooperative EGV-UAV control, the detailed set up of the simulation environment together with the generation of the 3D semantic map and the decision about the safety of the EGV and the 
evaluation of the results. The real demonstration scenarios will be refined and set up based on the simulation results and experiences.

\section{REFERENCES}

bobzilla, arkasha, and uhtu (2001-2020). Wigle.net All the networks. Found by everyone.

Cantelli, L., Mangiameli, M., Melita, C. D., and Muscato, G. (2013). UAV/UGV cooperation for surveying operations in humanitarian demining. In 2013 IEEE International Symposium on Safety, Security, and Rescue Robotics (SSRR), pages 1-6.

Casey C. Grant, P. and Merrifield, B. (2011). Analysis of Ambulance Crash Data. Technical report, Fire Protection Research Foundation.

CNN (2019). Car hit by train after avoiding crossing arm.

Cordts, M., Omran, M., Ramos, S., Rehfeld, T., Enzweiler, M., Benenson, R., Franke, U., Roth, S., and Schiele, B. (2016). The cityscapes dataset for semantic urban scene understanding. In Proc. of the IEEE Conference on Computer Vision and Pattern Recognition (CVPR).

Donoughe, K., Whitestone, J., and Gabler, H. C. (2012). Analysis of firetruck crashes and associated firefighter injuries in the united states. Annals of advances in automotive medicine. Association for the Advancement of Automotive Medicine. Annual Scientific Conference, 56:69-76.

Dosovitskiy, A., Ros, G., Codevilla, F., Lopez, A., and Koltun, V. (2017). CARLA: An open urban driving simulator. In Proceedings of the 1st Annual Conference on Robot Learning, pages 1-16.

fandom.com (2020). Halo alpha.

Forerunner Computer Systems (2020). Forerunner Computer Systems - Making Computers Simple.

Garmin (2020). Garmin forerunner 745 watch.

Harik, E. H. C., Guérin, F., Guinand, F., Brethé, J., and Pelvillain, H. (2015). UAV-UGV cooperation for objects transportation in an industrial area. In 2015 IEEE International Conference on Industrial Technology (ICIT), pages 547-552.

Inside Unmanned Systems (2019). One Ultra-Endurance LEAP for UAVs.

Lakas, A., Belkhouche, B., Benkraouda, O., Shuaib, A., and Alasmawi, H. J. (2018). A Framework for a Cooperative UAV-UGV System for Path Discovery and Planning. In 2018 International Conference on Innovations in Information Technology (IIT), pages 42-46.

Lee, J., Huang, R., Vaughn, A. F., Xiao, X., Hedrick, J., Zennaro, M., and Sengupta, R. (2003). Strategies of Path-Planning for a UAV to Track a Ground Vehicle. In In Proc. of the AINS 2003 conference.

NHTSA (2014). The National Highway Traffic Safety Administration and Ground Ambulance Crashes.

NOKIA (2016). 5G for Mission Critical Communication. Technical report, Nokia.
Peterson, J., Chaudhry, H., Abdelatty, K., Bird, J., and Kochersberger, K. (2018). Online Aerial Terrain Mapping for Ground Robot Navigation. Sensors, 18(2).

Smith, N. (2015). A National Perspective on Ambulance Crashes and Safety. Guidance from the National Highway Traffic Safety Administration on ambulance safety for patients and providers. EMS world, 44:912,94 .

Somogyi, H., Pup, D., Körös, P., Mihaly, A., and Soumelidis, A. (2018). Research of Required Vehicle System Parameters and Sensor Systems for Autonomous Vehicle Control. In 2018 IEEE 12th International Symposium on Applied Computational Intelligence and Informatics (SACI), pages 000027-000032.

Zoto, J., Musci, M. A., Khaliq, A., Chiaberge, M., and Aicardi, I. (2020). Automatic Path Planning for Unmanned Ground Vehicle Using UAV Imagery. In Berns, K. and Görges, D., editors, Advances in Service and Industrial Robotics, pages 223-230, Cham. Springer International Publishing. 